\title{
Don de gamètes, la fin de l'anonymat: la position britannique. Une position récente, dans le contexte européen
}

\author{
Françoise SHENFIELD
}

Dr Françoise Shenfield, médecin des hôpitaux, UCLH, membre du Taskforce Ethics and Law de l'ESHRE, et du comite d'éthique de la FIGO

\section{RÉSUMÉ}

L'anonymat du don de gamètes et d'embryons est entériné dans la loi française, mais non dans tous les pays européens. A l'heure où beaucoup de questions se posent sur "le droit à connaître ses origines" des enfants ainsi conçus, le récent abandon de cet anonymat en Grande Bretagne remet le sujet d'actualité. La chaîne d'évènement conduisant à la fin de l'anonymat depuis près de trois ans est décrite, comparée avec la position française et d'autres modèles internationaux, afin de réfléchir plus avant à notre responsabilité collective de professionnels de l'AMP, et aux conséquences possibles pour les familles ainsi créées.

Mots clés : anonymat, droit, origines, responsabilité

\section{INTRODUCTION}

Le don de gamètes, que ce soit de sperme ou d'ovocyte, est parfois la seule solution à la stérilité définitive de l'homme ou de la femme. La question du secret est débattue depuis nombre d'années, et quoique de plus en plus de couples décident de parler à leur enfant de leur origine "différente", par don de gamètes, l'anonymat des donneurs, inscrit dans la loi en France, est remis en question dans plusieurs pays [2]. En Grande Bretagne (GB) l'anonymat a été la règle jusqu'à fort récemment dans l'immense majorité des cas de don de sperme. Par ailleurs le don direct (entre personnes qui se connaissent), n'a jamais été interdit, et est surtout le fait du don d'ovocyte, jusqu'a $30 \%$ des cas. Outre Manche, (où exerce l'auteur), la pratique du don de gamètes se fait dans le cadre juridique du HFE Act 1990 [4], qui enjoint de prendre en compte l'intérêt ("welfare") de l'enfant à naître, et n'interdit le don ni à la femme célibataire, ni à celles en couple homosexuel.

Depuis environ 5 ans, s'est engagé en GB un questionnement sur la notion même d'anonymat, à la suite de débats similaires, notamment en Suède, aux Pays Bas et en Nouvelle Zélande [2] : fallait-il, dans l'intérêt de l'enfant surtout, faire connaître un jour à cet adolescent ou adulte l'identité du "donneur" de sperme ou de la donneuse d'ovocyte à l'origine de cet apport génétique, externe à celui de ses parents en droit. En effet, toutes les juridictions connues fonctionnent sur le même principe : c'est le couple « receveur », les parents " intentionnels » qui sont les parents légaux de cet enfant conçu par don de gamète, avec le consentement éclairé de toutes les parties, bien sûr. En conséquence, fallait-il que la loi impose le recrutement de donneurs qui s'engageraient à se faire connaître à l'enfant à sa "maturité" (majorité, 18 ans) comme le fait la loi suédoise depuis 1985 ?

\section{LA GRANDE BRETAGNE ET LE DON DE GAMĖTES: UN CHANGEMENT RADICAL AU 21 ème SIECLE}

En GB, la décision d'abandonner l'anonymat du don de gamètes et d'embryons, lui-même inscrit dans la loi française, a été remplacée par de nouvelles conditions, et entérinée le $1^{\mathrm{er}}$ avril 2005 [7]. Depuis cette date, les donneurs de gamètes ou d'embryons ne peuvent le faire que s'ils acceptent de révéler leur identité à l'enfant ainsi conçu lors de sa majorité, donc à cet "offspring", adulte issu du don. Cette décision fut

\section{Correspondance :}

Dr Françoise SHENFIELD - Reproductive medicine Unit, UCLH, Huntley street, LondresWC1 - Email mfi@easynet.co.uk 
prise après que la question ait fait l'objet de nombreux titres de presse, d'articles dans les revues médicales et psychosociales, ainsi que d'une campagne très concertée de la part de ce que l'on appelle dans les pays anglo-saxons des « groupes concernés " ou "lobbies". Cela signifie-t-il que le familial et le social sont en passe de se faire reléguer au deuxième plan par le génétique, ou simplement que le secret du don, pratiqué par plus de $80 \%$ des couples receveurs de don de sperme selon une étude multicentrique en Europe [8], se pratique moins et que l'accès aux origines se discute plus facilement dans nos sociétés ? Mais voyons d'abord comment s'est déroulé ce processus outre Manche.

Les premières pierres de ce changement important furent posées en 2001, lorsque le comité d'éthique de la Human Fertilisation and Embryology Authority (HFEA), l'agence de contrôle de l'Assistance Médicale à la Procréation (AMP) créée par la loi en 1991 [4], émit cette réflexion au sujet de l'anonymat et du don de gamètes : il s'agissait d'abord, "d'encourager l'ouverture dans l'intérêt de l'enfant", c'est à dire de décourager le secret ; néanmoins, rajoutait le comité "...la vie privée de la famille doit être respectée : donc... l'Etat ne peut ni ne doit forcer les parents à informer les enfants du don des circonstances de leur conception". Le comité ne fait qu'informer les membres de la HFEA au complet, qui prennent les décisions en son nom, lesquelles sont officialisées par l'inclusion dans le Code de Pratique [5] que doivent respecter toutes les cliniques pratiquant l'AMP. En fait, un nouveau courant s'annonçait, celui, "politiquement correct", arguant pour l'enfant du don "d'un droit à connaître ses origines".

En Janvier 2003, la ministre de la santé déclare, à l'occasion de la conférence annuelle de la HFEA qu'après «considération de tous les intérêts des différentes parties, il fallait partir du principe qu'il était juste que ces enfants ainsi conçus connaissent un jour l'identité du donneur". Le mode de transition n'était pas encore décidé, mais ce changement était clairement annoncé, et la décision de principe de la levée de l'anonymat éventuelle acceptée par le ministère, puisque son principe même était qualifié de «juste». II s'agissait alors seulement de continuer le débat sur la modalité et les étapes de ce changement radical.

De fait, depuis le HFE Act de 1990, l'identité des donneurs, et celle de tous les enfants de l'AMP, est inscrite dans un registre hyper-confidentiel tenu par la HFEA. De par la loi, les enfants du don ont le droit à des informations non identifiantes à l'âge de 18 ans, à condition bien sûr que leurs parents les aient informés de leurs " origines ». Le registre ayant été établi en 1991, ce n'est donc pas avant 2010 que les premiers enfants ainsi conçus sous le régime de la loi seront majeurs et qu'ils pourront obtenir ces informations non identifiantes, et s'enquérir de la probabilité d'épouser un demi-frère ou une demi-sœur génétique. II sera également possible d'obtenir d'autres informations, toujours alors non identifiantes : taille, poids, groupe ethnique, couleur des yeux et des cheveux, religion, intérêts, et un auto-portrait du ou des donneurs se décrivant en quelques lignes.

En 2001, et en prévision de ce changement qui allait créer des différences notables chez les enfants du don selon l'année de leur naissance, II fut aussi décidé de créer un « voluntary contact register 》 (un registre volontaire) dans lequel pourraient s'inscrire les donneurs, et les enfants/adultes conçus avant la loi de 1990 qui voudraient éventuellement prendre contact les uns avec les autres, et dont les données n'étaient pas sur le registre créé en 1991.

Depuis près de trois ans le changement radical voté en avril 2005 [7] instaure un nouveau système identifiant, de manière prospective évidemment : le nom et l'adresse, y compris la dernière adresse connue des donneurs et donneuses, pourront être communiqués à l'enfant, à sa majorité. C'est donc en 2023 que cette levée totale de l'anonymat deviendra opérationnelle, à condition encore une fois qu'ils aient été informés de leur origine par leurs parents.

Notons enfin que la situation est sans doute encore plus complexe pour le don d'embryon, car l'enfant à la recherche de ses origines pourrait aussi théoriquement retrouver des frères ou sœurs génétiques, plutôt que des demi frères ou sœurs (1/2 siblings). Notons aussi qu'en France on parle "d'accueil" d'embryon. Cette terminologie centrée sur la famille receveuse rappelle l'importance donnée a "l'intérêt (welfare) du futur enfant" inscrit dans la loi britannique [4], car il s'agit bien là de s'assurer que le couple destiné à " accueillir " cet embryon présente certaines garanties d'ordre médical ou psychologique. La différence est qu'en GB il ne s'agit pas de demander l'avis d'un juge, car c'est l'équipe soignante qui vérifie ces critères codifiés dans le Code de Pratique de la HFEA [5] , avec conseil psychologique et avis du médecin traitant si nécessaire. C'est alors à la HFEA, lors de ses inspections obligatoires d'un centre, de s'assurer que toutes les démarches recommandées par le Code de Pratique sont bien suivies.

\section{LA GESTION DU CHANGEMENT : FAITS CONNUS ET MODELLES}

Dans ce contexte mouvant, il est encore plus important d'agir de façon responsable, avec l'information la plus complète possible de nos patients. II s'agit en fait de leur dire tout ce que nous savons, mais aussi ce que nous ne savons pas, en s'appuyant sur les quelques études publiées. Tout en entendant l'angoisse exprimée publiquement par quelques adultes [12] à la recherche de l'identité du géniteur qui a donné son sperme à leur parents, sachons que les études publiées provenant de la Suède, qui a précédé la GB en abandonnant l'anonymat du don en 1985 [2], sont pour l'instant encore focalisées sur l'attitude parentale. Deux études rapportent le suivi de parents suédois, et analysent ce qui est "dit" dans ces familles. En 2002, celle de Gotlieb et al. [9] montre que moins de $50 \%$ des enfants avaient été informés de leurs origines par leur parents depuis la loi de 1985. La dernière étude, publiée en 2007 [10] par le même groupe, interroge 19 couples de parents : $61 \%$ avaient informé leur enfant de leur origine par don de sperme, mais moins des $2 / 3$ avaient en fait spécifié qu'il pourrait un jour connaître le nom du donneur.

Notons donc que ces études analysent le parcours des parents receveurs de don, mais non celle des enfants du don, et qu'il s'agit de familles receveuses d'un don de sperme, car le don d'ovocyte n'a été permis en Suède que depuis trois ans. 


\section{EVOLUTION RÉCENTE DU NOMBRE DES DONS EN GRANDE BRETAGNE}

Quelles sont les conséquences pratiques du changement récent en GB ? La HFEA avait clairement souligné que "la chute prévue du nombre de donneurs", contre-argument donné par de nombreuses équipes médicales [11] pratiquant le don à la levée de l'anonymat, "n'était pas une raison suffisante pour interdire aux enfants ainsi conçus de connaître leur identité".

Le Tableau 1 [6] montre qu'il y a eu en effet un déclin du nombre total de donneurs de sperme entre 2000 et 2005 , avec une certaine recrudescence en 2006, pour le don de sperme, mais non celui d'ovocytes. Les optimistes qui espèrent que de nouveaux donneurs, prêts à être identifiés 18 à 19 ans plus tard, seront recrutés auront peut être raison, mais il est trop tôt pour l'affirmer, chiffres à l'appui.

Pour ce qui est du don d'ovocyte, la chute est d'autant plus marquée que le nombre était relativement élevé. Notons par ailleurs que la proportion de "partage d'ovocytes" (calculée seulement depuis 3 ans) est d'environ $50 \%$ des dons d'ovocytes. Ce système permet en GB (ainsi qu'au Danemark et en Israël) aux femmes faisant un cycle de FIV "d'échanger" un certain nombre d'ovocytes contre une compensation qui diminue le prix du cycle dans le secteur privé. Cette solution pragmatique autorisée par la HFEA à la fin des années 90 , présente le danger évident de coercition possible des couples qui n'ont pas les moyens financiers de pourvoir au coût de la FIV. De plus, on imagine le dilemme de celles qui "partagent" et pourraient apprendre à l'avenir qu'elles sont restées sans enfants et que leur receveuse est mère grâce à elles. Tout cela bien sûr est expliqué en détail par les psychologues chargés de l'information des donneurs ("implication counselling"). Sachons enfin que le système britannique est loin d'être l'un des plus généreux d'Europe, avec seulement $30 \%$ des cycles de FIV pratiqués dans le National Health Service, et que bien sûr un accès plus équitable, maintes fois promis par le ministère de la santé, permettrait aux couples qui ont la générosité de faire ce partage de l'envisager sans raison pécuniaire.

\section{CONCLUSION}

Au fils des ans le secret du don est sans doute devenu moins fréquent et le sujet " stérilité "n'est plus aussi tabou. Mais la levée de l'anonymat, toujours sujette à l'information des parents, pose les questions du quand, comment, et surtout de ce qui est "mieux" pour l'enfant. Il est évident que nul ne sait comment vont se passer les contacts entre donneurs et enfants du don. Même si le modèle de l'adoption offre certaines leçons exemplaires - "dire" aux enfants leur origine le plus tôt possible est sans doute la meilleure approche - nous pensons que les enfants du don sont désirés par leurs parents bien avant leur naissance [13], et que ce modèle ne peut donc être transposé qu'imparfaitement. Le plus important pour les familles receveuses du don est sans aucun doute le support psychologique qui doit être facile d'accès pour toutes les personnes concernées durant les étapes successives d'un parcours qui sera sans doute complexe, même s'il ne concerne qu'une minorité de tous les actes d'AMP.

En ce qui nous concerne, les spécialistes de l'AMP, nous pratiquons en GB dans le cadre juridique du HFE Act 1990, qui nous enjoint de prendre en compte l'intérêt ("welfare") de l'enfant, sans plus de précision que le guide du Code de Pratique (Code of Practice, COP) de la HFEA. Il me semble par ailleurs évident, en tant que praticien, que l'intérêt de cette future personne vulnérable est aussi partiellement notre responsabilité (avec celle des futurs parents), mais peut être encore plus importante moralement que celle que nous devons en droit et en déontologie à nos patients (couple ou femme célibataire, puisque cela n'est pas interdit en GB) en raison même de cette vulnérabilité de l'enfant.

La question la plus importante serait donc : qu'en est-il de l'intérêt de l'enfant issu du don ? Et la réponse, que nous ne sommes pas persuadés qu'un système, anonymat ou non, est préférable à l'autre. De fait, en France, le CCNE [1] s'est prononcé pour le maintien de l'anonymat, et a rejeté la «double voie», où les couples auraient la possibilité de choisir des gamètes issus d'un

Tableau 1 : Nombre de nouveaux donneurs de gamètes annuel en GB depuis 2000 [6].

\begin{tabular}{lcccc}
\hline Année & $\begin{array}{c}\text { Donneurs de } \\
\text { sperme }\end{array}$ & Année & $\begin{array}{c}\text { Donneuses } \\
\text { d'ovocytes }\end{array}$ & $\begin{array}{c}\text { Donneuses } \\
\text { Année } \\
\text { d'ovocytes partagés } \\
\text { donneuses dans le total des }\end{array}$ \\
\hline 2000 & 325 & 2000 & 1242 & \\
2001 & 328 & 2001 & 1315 & \\
2002 & 278 & 2002 & 1179 & 502 \\
2003 & 255 & 2003 & 1056 & 410 \\
2004 & 247 & 2004 & 1064 & 317 \\
2005 & 260 & 2005 & 956 & 04 \\
2006 & 295 & 2006 & 768 & 06 \\
\hline
\end{tabular}


don anonyme ou non anonyme. Cette double voie fut abandonnée aux Pays Bas, mais c'est celle que le groupe de réflexion Ethique et Droit de I'ESHRE recommande dans ses considérations sur le don de gamètes [3].

La situation est donc très évolutive, et l'on se demande que sera l'avenir pour les enfants du don en Europe, et leur réaction ainsi que celle de leur famille, et celle des donneurs - à la rencontre éventuelle lors de leur majorité. Nous savons que le secret peut être ressenti comme un non dit entre parents et enfant, comme le souligne le rapport du CCNE [1] sur la question de l'anonymat : (il faut savoir) "les conséquences éventuellement délétères du point de vue psychologique qui peuvent résulter d'une absence d'information des enfants sur les modalités de leur conception, et plus encore de leur installation dans un système de mensonge familial".

Pourtant, le paradoxe est là : s'il n'y a plus de secret sur l'identité des donneurs, les parents choisiront peut être plus souvent le secret des origines, afin de préserver leur "vie privée", et le cercle familial. Sans oublier la responsabilité que nous avons aussi vis à vis des généreux donneurs de gamètes, sachons faire part de nos incertitudes, et soyons clairs : une expérience psychosociale est en train de se construire, dont seules quelques données sont connues malgré le précédent suédois. Ce sujet est loin d'être clos.

\section{RÉFÉRENCES}

1. CCNE : Avis 90 , accès aux origines, anonymat et secret de la filiation.

2. DANIELS K. : Donor gametes : anonymous or identified ?, Best Practice Res. Clin. Obstet. Gynaecol., 2006 : 21, 1 : 113-128.

3. ESHRE Ethics and law taskforce 3. Shenfield F., Pennings G., Sureau C., Cohen J., Devroey P., Tarlatzis B. : Gametes and Embryo donation. Hum. Reprod., 2002, 17 : 1407-1408.

4. HFE Act 1990. HMSO.

5. HFEA Code of Practice, http://www.hfea.gov.uk.

6. HFEA Data: $h$ htp://www.hfea.gov.uk/d ocs/Latest_long_term_data_ analysis_report_front_cover.pdf.

7. Loi : http://www.opsi.gov.uk/si/si2004/20041511.htm.

8. GOLOMBOCK S., BREAWAYS A., COOK R. et al. : The European study of assisted reproduction families : family functioning and child development. Hum. Reprod., 1996, 11 : 2324-2331.

9. GOTTLIEB C., LALOS O., LINDBLAD F. : Disclosure of donor insemination to the child : the impact of Swedish legislation on couple's attitudes. Hum. Reprod., 2000, 15, 9 : 2052-2056.

10. LALOS O., GOTTLIEB C. : Legislated right for DI children to know their genetic origin, a study of parental thinking. Hum. Reprod., 2007, 22 : 1759-1768.

11. PAUL S., HARBOTTLE S., STEWART J.A. : Recruitment of sperm donors : the Newcastle experience 1994-2003. Hum. Reprod., 2006, $21: 150-158$.

12. Rose and another $v$ Secretary of Sate for Health and HFEA, 2002, EWHC 1593, case no: CO/3802/01.

13. SHENFIELD F:, Privacy versus disclosure in gametes donation:a clash of interests, of duties, or an exercise in responsibility ? J. Assist. Reprod. Genetics, 1997, $14: 369-371$.
Manuscrit reçu : janvier 2008 ; accepté mars 2008.

Communication présentée lors du XXIVème Congrès de la SALF, Colmar décembre 2007.

\author{
ABSTRACT \\ Gametes donation: the end of anonymity and its \\ consequences
}

Françoise SHENFIELD

Although anonymity is enshrined in law in France, this is far from the case in all European countries. It has been relinquished since 1985 in Sweden and more recently in the UK.

The transition phase in the UK is highlighted, and available evidence on the effects on the number of donors analysed, as well as the ethical and legal background for donors, recipients, and offspring.

Key words : anonymity, ethics, gametes donation, law, offspringwelfare 